\title{
Una aproximación a la Conjetura de Goldbach
}

\author{
An Approach to the Goldbach Conjecture

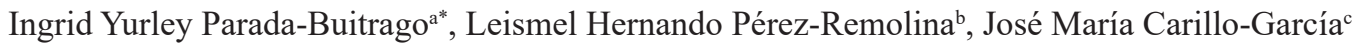 \\ a*Licenciada en matemáticas e informática, faithwhite24@hotmail.com, Institución educativa Colegio Santos Apóstoles, https://orcid. \\ org/0000-0003-3563-5965, Villa del Rosario, Colombia \\ bLicenciado en matemáticas e informática, lerx555@hotmail.com, Institución educativa Colegio Integrado Fe y Alegría, \\ https://orcid.org/0000-0003-1570-8653, Villa del Rosario, Colombia \\ 'Ingeniero Industrial, Josmacg@gmail.com, Centro Educativo Rural Agua Blanca, https://orcid.org/0000-0001-5902-5710, Cúcuta, \\ Colombia
}

Forma de citar: Parada-Buitrago, I.Y. Pérez-Remolina, L.H. Carillo-García, J.M. Una aproximación a la Conjetura de Goldbach. Eco Matemático, 10 (1), 104-113

Recibido: 20 septiembre 2018

Aceptado: 5 diciembre 2018

\section{Palabras clave \\ Conjetura Goldbach, números primos, demostración, análisis.}

Resumen:Uno de los problemas de matemáticas que parece muy cierto, pero carece de una demostración es la conjetura de Goldbach planteada desde 1742.El presente artículo realiza un análisis crítico, donde se recopiló diversas propuestas de solución de ésta, con un leguaje y una nomenclatura unificada para qué sea asequible al estudioso de la matemática. Es una investigación histórica y exploratoria de tipo cualitativa que concluye con una visión holística, que permita identificar los intentos de demostración más relevantes de la Conjetura de Goldbach, de esta manera se pretende estimular al estudiante a desarrollar su capacidad investigativa hacia las Matemáticas, evidenciando: los posibles métodos de investigación en el área, la aparición de teorías y los nuevos desafíos para los matemáticos. Aquí se presentaron intentos de demostración a partir de la teoría de números primos, en la búsqueda de fórmulas que describan todos los primos, intento que nunca se ha logrado concretar. Demostraciones que echan mano de la probabilidad con que aparecen los números primos, que nuevamente son esquivos a dejarse encasillar. Se echó mano a la lógica en otra de los intentos aquí presentados, nuevamente sin el éxito esperado. Divide y vencerás tal vez pensó Ricardo G. Barca en el intento que aquí se presenta, pero se le escapó alguna parte. Siguiendo la idea de Polya, se trata de solucionar siguiendo el camino que ha dado solución a otros problemas mediante un acercamiento heurístico, pero sin lograrlo.

\footnotetext{
*Autor para correspondencia: faithwhite24@hotmail.com
} 


\section{Keywords \\ Goldbach's conjecture, prime numbers, demonstration, analysis.}

\section{Introducción}

Cuando se admira una obra creada por el hombre, sea ella una pintura, escultura un edificio, una formula química o un resultado matemático, en general se piensa en la mente brillante que la creo, es posible aun que se recuerde su nombre, no se podrá nombrar la capilla Sixtina sin recordar el genio creador de Miguel Ángel y si se piensa en triángulos la mente traerá a Pitágoras.

Se puede pensar que la humanidad se ha desarrollado gracias a estos iluminados, o pensaran algunos a seres extraterrestres que los inspiraron de alguna manera, pero la cosa no es así, bien decía Newton parafraseando a Bernard de Chartes: "si he logrado ver más lejos ha sido porque he subido a hombros de gigantes" (Newton, 1676) y aquí empieza un proceso largo y tortuoso, el de la construcción o en matemáticas el de la demostración, este proceso puede durar años y en algunos casos siglos de intentos ligeramente exitosos o simplemente fracasos, pero cuando al fin se logra lo acometido, se olvida el proceso y se aplica un teorema que se dice que es de algún Tales de Mileto, se navega a través del canal de Panamá o se admiran las Pirámides con alguien contando una historia interplanetaria.

El presente trabajo consiste en realizar un análisis a la Conjetura de Goldbach, la cual es un problema matemático enmarcado en la Teoría de Números y en la que los estudiosos de la matemática centran su atención desde hace muchos años. La Conjetura de Goldbach (1742) se resume en: todo número par, mayor que 2 se puede escribir como la suma de dos números primos.

El problema radica en el hecho de no conocer el proceso de construcción que ha desaparecido con la obra terminada y con sus constructores desaparecidos. Los astrónomos se emocionan hasta el delirio cuando pueden rastrear el nacimiento de una estrella. De esto se trata el trabajo de 
investigación que se pretende desarrollar, hay un resultado matemático en proceso de construcción, además con mucha documentación, de antes de la inspiración durante el proceso mismo de inspiración con las cartas entre Euler, Waring y Goldbach, que entre otras cosas dejan claro que la inspiración, como se ha afirmado anteriormente, no es de una sola persona, pero al fin se le debe atribuir alguna y esta estrella en construcción es precisamente la Conjetura de Goldbach, como el proceso no ha terminado, a pesar que en él han trabajado, por ya casi tres siglos, las mentes más brillantes de la matemática.

Se busca que por medio de la observación del proceso en ciernes de la conjetura de Goldbach, mostrar cómo es el trabajo de la construcción de los resultados de las matemáticas, obra de hombres que se esfuerzan, se equivocan, crean herramientas, pero ante todo perseveran en la búsqueda de la satisfacción de encontrar la verdad. Y de esta manera se espera inspirar a toda persona interesada en esta área, en el trabajo de crear matemáticas.

La gran inquietud es como desarrollar un documento que contribuya a la inspiración de los estudiosos de la matemática, se parte de una síntesis que se hace a la documentación que desde el punto de vista de los autores es la más relevante en el estudio de la Conjetura de Goldbach, la información está escrita de forma general por este motivo se hace indispensable integrar las teorías necesarias en un lenguaje accesible, el resultado es la elaboración de un documento que presente en un orden de complejidad y cronológico los esfuerzos hasta el momento realizados en busca de una demostración de la Conjetura de Goldbach.

El documento se planea de la siguiente manera: en un primer capítulo, se hace una introducción al problema en el cual se plantea, formula y justifica el problema sobre la Conjetura de Goldbach, además se establecen los objetivos y los alcances que presentara el documento; en el segundo capítulo, se presentaran antecedentes que se relacionan con el desarrollo del trabajo, acompañado de las teorías que se hacen necesarias para el desarrollo del trabajo y un marco legal para enmarcar el trabajo realizado; en un tercer capítulo, se establece la metodología usada para obtener los resultados de la investigación : en un cuarto capítulo, se establecen dos subcapítulos; en el primer subcapítulo, Se postula la Conjetura de Goldbach contextualizando el problema, situando el lector en la época donde se desenvuelven los personajes que marcan el inicio del problema; En un segundo subcapítulo, se hará un análisis de las principales seudodemostraciones y avances hasta donde el resultado fue correcto, se presentara finalmente, en el capítulo de conclusiones, se destacara los principales errores en los cuales se han encontrado contradicciones que han imposibilitado la solución a la Conjetura de Goldbach.

Al finalizar la lectura del documento, el lector tendrá los fundamentos para la comprensión de los intentos más relevantes de solución que se han propuesto a la Conjetura de Goldbach e iniciar un camino a una posible demostración de un problema matemático que se ha mostrado esquivo durante la historia.

\section{Materiales y métodos}

Se hará uso de la investigación cualitativa, en la cual se debe comprender su concepción desde el punto de vista de algunos autores.

Para Hernández (2012), no es el estudio de cualidades individuales e independientes; contrario a esto, ella, corresponde a un estudio integrado y por tanto constituye unas unidades de análisis. Rueda (2007), la define como como un proceso en donde intervienen varias visiones que tienen como objetivo dividir los fenómenos y determinar las relaciones que existen entre sus componentes. Finalmente, para González (2013), la investigación cualitativa tiene como propósito la construcción de conocimiento sobre la realidad social, a partir de las 
condiciones particulares y la perspectiva de quienes la originan y la viven.

Se hará uso de los siguientes instrumentos de análisis de información:

Las fichas bibliográficas, donde se identifica el título, autor, hipótesis, propósito, entre otras; que orientan de una manera más sencilla la elaboración de una síntesis analítica y critica que contribuya en la interpretación de manera idónea sobre el objeto de estudio.

\section{Resultados y discusiones}

\section{Postulación de la conjetura}

Luego de mantener una extensa correspondencia el 7 de junio de 1742 Goldbach, que se encontraba en Prusia escribe a Euler que se encontraba en Moscú sobre la utilidad de que "se hagan proposiciones que son muy probables, incluso si no están aún sustentadas por una demostración, pues aunque con el tiempo se demuestre que son incorrectas pueden contribuir a descubrir una nueva verdad" y a continuación se aventura a proponer "todo número que está compuesto [como suma] de dos números primos es a la vez la suma de tantos números primos como queramos (incluyendo 1), hasta llegar a la suma que consiste sólo de unos" a la que Euler (1742) responde el 30 de junio del mismo año que "Sin embargo que todo número par sea la suma de dos números primos, lo que considero un teorema correcto, es algo que no puedo demostrar" dando origen a la llamada conjetura de Goldbach.

\section{Ricardo G. Barca - Universidad Tecnológica Nacional}

La solución presentada es incompleta, pues trata de probar tres casos y solo hace la prueba para dos de estos casos, los cuales a través de observación se evidencia su veracidad; demuestra para números primos $\leq 86$, luego con esto no se prueba nada en la conjetura de Goldbach debido a la corta cantidad de números tomada.

El autor plantea un camino que deja incompleto por tanto el trabajo realizado no alcanza el objetivo propuesto, además las consideraciones que se han hecho están basadas en cortos grupos de números, imposibilitando plantear un camino correcto que lleve a la solución de la Conjetura de Goldbach.

\section{Ciencia y Lógica son suficientes}

El autor inicia explicando detalladamente la notación lógica utilizada, aunque el documento original usa dos tipos de notación, en este caso se ha editado para unificar a una sola notación.

La demostración es realizada mediante notación lógica, por lo cual el autor inicia indicando la escritura en notación lógica de la Conjetura de Goldbach, luego propone una demostración por método de absurdo, por lo cual se hace una negación a la escritura de dicha conjetura y se hace una explicación del proceso y la veracidad de esta negación.

El documento está distribuido mediante una numeración que va hasta el numero 20 donde en cada una se hace una propuesta que se comprueba, sirviendo para las próximas proposiciones para formar un camino que lleve a la demostración.

El camino formado para la demostración presenta fallas, los cuales se presentan a continuación:

En el inciso 6, se plantea, $\forall p \forall q \exists s(N(p) \wedge N(q) \wedge M(s) \wedge q=p+s)$, es verdadera.

$$
M(x) \text { dice « } x \text { es entero no negativo». }
$$

Según lo anterior la condición que para todo $\mathrm{p}$ y q existe un $\mathrm{s}$, tal que $\mathrm{p}$ y q son números naturales y $\mathrm{s}$ es un numero entero no negativo, a partir de esto se establece la condición $q=p+s$ donde q es 
necesariamente mayor que $\mathrm{p}$ a menos que $s=0$; el problema en este planteamiento es que está incompleto para ser verdadero y el autor dice "no es especificado por ser trivial" lo que lleva a formar un planteamiento incorrecto.

Seguidamente el autor toma este planteamiento errado para justificar posteriores afirmaciones, tal como se muestra a continuación:

$$
" \forall p \forall q \exists s(P(p) \wedge P(q) \wedge M(s) \wedge q=p+s)
$$

La demostración está basada en un camino que el autor construye a través de 23 incisos los cuales se complementan unos a otros en orden ascendentes, por lo tanto, a partir del de la demostración errada que se hace en el inciso 6 la demostración pasa a ser incorrecta a partir de allí, pues tal como se observa el inciso 6 es vital para la solución del inciso 8 y así sucesivamente, por lo tanto, la conclusión a la cual el autor llega pierde su validez.

\section{Rafael Tesoro - Acercamiento heurístico}

Si se considera un número par $N$ y se toma al azar un número $n \leq N$ y se llama $B$ al suceso "n es primo". La probabilidad (P) de este suceso depende de $N$.

Ejemplo. $\mathrm{N}:=4 \mathrm{Si}$ se tiene en cuenta la probabilidad de escoger un numero primo menor o igual a 4 es de $1 / 2=0.5$ pues hay 2 números primos ( 2 y 3), pero si se considera un número $\mathrm{N}$ más alto se puede apreciar que la probabilidad disminuye; $\mathrm{N}:=10$ la probabilidad es de $4 / 10=0.4$ por lo tanto a medida que se aumenta $\mathrm{N}$ la probabilidad disminuye.

Si dicha probabilidad se considera uniforme se toma la función $\pi(\mathrm{x})$ y por el teorema de los números primos la formula asintótica es

$$
\mathbb{P}(\mathrm{B})=\frac{\pi(\mathrm{N})}{\mathrm{N}} \sim \frac{1}{\log \mathrm{N}}
$$

Siendo $\pi(\mathrm{N})=\#\{\mathrm{p} \leq \mathrm{N}: \mathrm{p}$ primo $\}$

$\mathrm{Si}$ se llama $\mathrm{C}$ al suceso "N-n es primo" la probabilidad de $\mathrm{C}$ es aproximadamente igual a

${ }^{1} \log ^{\mathrm{N}} \mathrm{Y}$ a $\mathrm{P}(B)$, entonces

$$
\mathbb{P}(\mathrm{C}) \sim \frac{1}{\log \mathrm{N}} \sim \mathbb{P}(\mathrm{B})
$$

Si se imagina que dicha distribución es uniforme y con cierto grado de aproximación hacia n del mismo orden de $N y$ que son estos n los que contribuyen mas al numero de representaciones de $\mathrm{N}$ como suma de dos primos $\left(\mathrm{r}_{2}(N)\right)$. Es por ello que se asume la hipótesis de que $\mathbb{P}(\mathrm{C}) \sim \frac{1}{\log N} \sim \mathbb{P}(\mathrm{B})$

Luego se hace la pregunta ¿se puede estimar asintóticamente el valor de

$$
\begin{aligned}
& \left.\mathrm{r}_{2}(\mathrm{~N}):=\mathbb{\#}\left(\mathrm{p}_{1}, \mathrm{p}_{2}\right) \in \mathcal{P} \times \mathcal{P} \mid \mathrm{N}=\mathrm{p}_{1}+\mathrm{p}_{2}\right\} \quad \text { Como } \frac{\mathrm{r}_{2}(\mathrm{~N})}{\mathrm{N}}=|\mathrm{B} \cap \mathrm{C}| * \frac{1}{\mathrm{~N}}=\mathbb{P}(\mathrm{B} \cap \mathrm{C})=\mathbb{P}(\mathrm{B}) * \\
& \mathbb{P}(\mathrm{C}) \sim \frac{1}{\left.(\log )^{2}\right)^{2}} ?
\end{aligned}
$$

La respuesta es negativa debido a la dependencia entre la condición que n sea primo y que $N-n$ también lo sea, como consecuencia se incumple la igualad de $\boldsymbol{P}(B \cap C)=P(B) * \boldsymbol{P}(C)$. Colocamos un ejemplo en el cual se evidencie la dependencia en el caso de que $\mathrm{n}$ sea un primo de $\mathrm{N}$ entonces la correlación es total entre $p|n y p| N-n$.

Ejemplo. Sea $N:=16$

$$
\begin{gathered}
B: \quad n \in\{2,3,5,7,11,13\} \quad C: \quad n \in\{3,5,11,13\} \\
\mathbb{P}(B \cap C)=\frac{4}{16} \neq \frac{6}{16} * \frac{4}{16}=\mathbb{P}(B) * \mathbb{P}(C)
\end{gathered}
$$

EL suceso $B$ depende del suceso $C$ y viceversa. Partimos de $\mathrm{P}(B \cap C)=\left(\mathrm{r}_{2}(\mathrm{~N})\right) \mathrm{N}$

$$
\begin{gathered}
\frac{\mathbb{P}(B) * \mathbb{P}(C)}{\mathbb{P}(B) * \mathbb{P}(C)}=1 \\
\Rightarrow \mathbb{P}(B \cap C)=\frac{r_{2}(N)}{N}=\frac{\mathbb{P}(B) * \mathbb{P}(C)}{\mathbb{P}(B) * \mathbb{P}(C)} * \mathbb{P}(B \cap C) \\
\mathbb{P}(B \cap C)=\frac{r_{2}(N)}{N}=\mathbb{P}(B) * \mathbb{P}(C) * \frac{\mathbb{P}(B \cap C)}{\mathbb{P}(B) * \mathbb{P}(C)}
\end{gathered}
$$




$$
\begin{gathered}
\text { Como } \mathbb{P}(\mathrm{C}) \sim \frac{1}{\log N} \sim \mathbb{P}(\mathrm{B}) \quad \text { Entonces } \\
\mathbb{P}(\mathrm{B} \cap \mathrm{C})=\frac{\mathrm{r}_{2}(\mathrm{~N})}{\mathrm{N}} \sim \frac{1}{\log \mathrm{N}} * \frac{1}{\log \mathrm{N}} * \frac{\mathbb{P}(\mathrm{B} \cap \mathrm{C})}{\mathbb{P}(\mathrm{B}) * \mathbb{P}(\mathrm{C})} \\
\mathbb{P}(\mathrm{B} \cap \mathrm{C})=\frac{\mathrm{r}_{2}(\mathrm{~N})}{\mathrm{N}} \sim \frac{1}{(\log \mathrm{N})^{2}} * \frac{\mathbb{P}(\mathrm{B} \cap \mathrm{C})}{\mathbb{P}(\mathrm{B}) * \mathbb{P}(\mathrm{C})}
\end{gathered}
$$

Multiplicamos por $N$ a ambos lados y nos resulta la ecuación inicial.

$$
\mathrm{NP}(\mathrm{B} \cap \mathrm{C})=\mathrm{r}_{2}(\mathrm{~N}) \sim \frac{\mathrm{N}}{(\log \mathrm{N})^{2}} * \frac{\mathbb{P}(\mathrm{B} \cap \mathrm{C})}{\mathbb{P}(\mathrm{B}) * \mathbb{P}(\mathrm{C})}
$$

Se llama ahora $\rho(\mathrm{N}) \mathrm{Al}$ factor

Al cual se aproximó y suponem $\frac{\mathbb{P}(B \cap C)}{\mathbb{P}(B) * \mathbb{P}(C)}$ rategia para acercarnos.

- Sustituir el suceso $B$ (n es Primo) por una cantidad de sucesos más elementales en las cuales se considera que $n$ no sea divisible por un numero primo $p$.

- Hacer lo mismo para el suceso $C$.

- Acumular las probabilidades elementales multiplicándolas.

Iniciamos reemplazando la condición $\mathrm{B}$ (n es primo) con la condición $B_{p}$ En la cual $n$ no sea divisible por todos los primos menores que $\sqrt{ } \mathrm{N}$, llevando a que $\mathrm{n}$ no sea múltiplo de un primo $\mathrm{p}$ (lo mismo con el suceso $\mathrm{C}_{\mathrm{p}}$ ). Ahora se estima que:

$$
\rho_{p}(N)=\frac{\mathbb{P}\left(B_{p} \cap C_{p}\right)}{\mathbb{P}\left(B_{p}\right) * \mathbb{P}\left(C_{p}\right)}
$$

Se observa que $\mathrm{P}\left(\mathrm{B}_{\mathrm{p}}\right)=1-\mathrm{P}\left(\mathrm{B}_{\mathrm{p}}\right)^{\mathrm{c}}$, para calcular los números múltiplos de un número (en este caso primo $\mathrm{p}$ ) se toma la función parte entera $[\mathrm{N} / \mathrm{p}] \mathrm{Y}$ para calcular la probabilidad de este evento se multiplica $[\mathrm{N} / \mathrm{p}]^{* 1} / \mathrm{N}$
Ejemplo. Dado en número $\mathrm{N}:=20$ se quiere saber los números múltiplos de 3 que estén por debajo de 20; entonces:

$[\mathrm{N} / \mathrm{p}]=[20 / 3]=[6,66 \ldots]=6$

Y para calcular esta probabilidad multiplicamos este resultado por $1 / \mathrm{N}=1 / 20$

$$
6 * 1 / 20=0,3
$$

Retomando lo anterior se afirma que $P\left(B_{p}\right)=1$ $P\left(B_{p}\right)^{c}=1-[N / p]^{* 1} / N Y$ aproximadamente igual $(\sim)$ a $1-1 / \mathrm{p}$, no es igual debido al uso de la función parte entera. Por simetría se dice que $P\left(C_{p}\right)=P\left(B_{p}\right)$.S hace la pregunta $i$ se puede calcular $P\left(B_{p} \cap C_{p}\right)$ ?

A continuación se examinan los dos casos posibles en los cuales $p \mid n y p / n$.

Caso 1. p|n. Se tiene que $\mathrm{B}_{\mathrm{p}}=\mathrm{C}_{\mathrm{p}}$ Porque $N-n$ es múltiplo de $\mathrm{p}$ si y solo si n es múltiplo de $p$, entonces

Como $B_{p}=C_{p} \Rightarrow \mathbb{P}\left(B_{p}\right)=\mathbb{P}\left(C_{p}\right)$ Lo que nos lleva a

$$
\rho_{p}(N)=\frac{\mathbb{P}\left(B_{p} \cap C_{p}\right)}{\mathbb{P}\left(B_{p}\right) * \mathbb{P}\left(C_{p}\right)}=\rho_{p}(N)=\frac{\mathbb{P}\left(B_{p} \cap C_{p}\right)}{\mathbb{P}\left(B_{p}\right)^{2}}
$$

Ahora como $B_{p}=C_{p} \Rightarrow \mathbb{P}\left(B_{p}\right)=\mathbb{P}\left(C_{p}\right)$ Como son iguales y la probabilidad es la misma $\mathbb{P}\left(B_{p}\right)=\mathbb{P}\left(B_{p} \cap C_{p}\right)=\mathbb{P}\left(C_{p}\right)$, llegando

$$
\begin{gathered}
\qquad \rho_{p}(N)=\frac{\mathbb{P}\left(B_{p} \cap C_{p}\right)}{\mathbb{P}\left(B_{p}\right)^{2}}=\frac{1}{\mathbb{P}\left(B_{p}\right)} \\
\text { Teniendo en cuenta que } \mathbb{P}\left(B_{p}\right) \sim 1-\frac{1}{p} \text { Entonces } \\
\frac{1}{\mathbb{P}\left(B_{p}\right)} \sim \frac{1}{1-\frac{1}{p}}=\frac{1}{\frac{p-1}{p}}=\frac{p}{p-1} \\
\frac{p}{p-1}=\frac{p+1-1}{p-1}=\frac{p-1}{p-1}+\frac{1}{p-1}=1+\frac{1}{p-1}
\end{gathered}
$$

Por lo tanto

$$
\rho_{p}(N)=\frac{\mathbb{P}\left(B_{p} \cap C_{p}\right)}{\mathbb{P}\left(B_{p}\right)^{2}}=\frac{1}{\mathbb{P}\left(B_{p}\right)} \sim \frac{p}{p-1}=1+\frac{1}{p-1}
$$

Caso 2. $\mathrm{p} / \mathrm{N}$. Ahora deber ser $\mathrm{p}>2$ debido a que $\mathrm{N}$ es par; la condición dice que $\mathrm{p}$ no divide a $N$ y 2 divide a todo numero par. Se enumeraran clases residuales modulo $p$. Sea $r_{N}>0$ el residuo de dividir $N$ por p. El número n puede estar en el residuo (Clases Residuales) con probabilidad con probabilidad 1/p (por hipótesis). EL suceso $B_{p}$ Es la unión de ocasiones en que el residuo de $\mathrm{n}$ es 
congruente con $\mathrm{r} \_\mathrm{n}$ Para modulo $\mathrm{p}, \mathrm{n} \equiv \mathrm{r}_{\mathrm{n}}(\bmod p)$ que esta entre $1,2, \ldots, \mathrm{p}-1$ y entonces $P\left(B_{p}\right)=\left(p^{-}\right) / p$. También por distribución uniforme $P\left(C_{p}\right)=\left(p-{ }^{1}\right) / p$. El suceso $\left(B_{p} \cap C_{p}\right)$ Equivale a $r_{\mathrm{n}} \in\{1, \ldots, \mathrm{p}-1\}-\left\{\mathrm{r}_{N}\right\}$.

Ejemplo. Sea $\mathrm{N}:=14$ y sea $\mathrm{p}:=5$

Ahora evaluamos los sucesos $B_{p}$ (n no es múltiplo de un primo p) y $C_{p}(N-n$ no es múltiplo del primo de $p$.

$$
\begin{aligned}
& B_{5}=\{1,2,3,4,6,7,8,9,11,12,13,14\} \\
& C_{p}=\{0,1,2,3,5,6,7,8,10,11,12,13\}
\end{aligned}
$$

En $B_{p}$ Se excluye a $\{0,5,10\}$ Por ser múltiplos de 5 y en $\mathrm{C}_{\mathrm{p}}$ Es excluye a $\{4,9,14\}$ Porque al aplicar N-n resultan múltiplos de 5 .

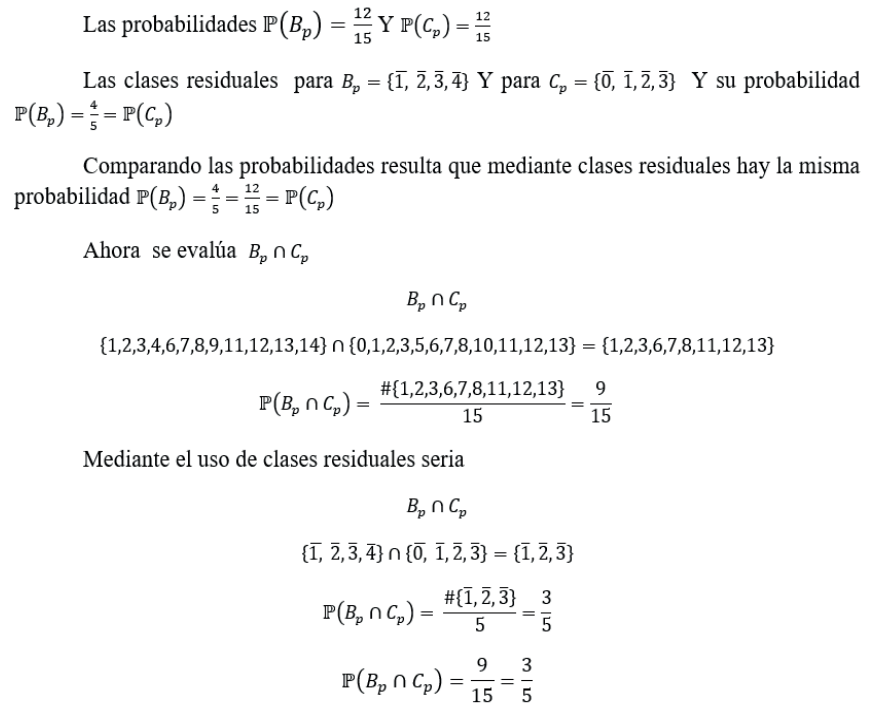

Comparando las probabilidades resulta que mediante clases residuales hay la misma probabilidad $\mathbb{P}\left(B_{p}\right)=\frac{4}{5}=\frac{12}{15}=\mathbb{P}\left(C_{p}\right)$

Ahora se evalúa $B_{p} \cap C_{p}$

$$
B_{p} \cap C_{p}
$$

$\{1,2,3,4,6,7,8,9,11,12,13,14\} \cap\{0,1,2,3,5,6,7,7,8,10,11,12,13\}=\{1,2,3,6,7,8,11,12,13\}$$$
\mathbb{P}\left(B_{p} \cap C_{p}\right)=\frac{\#\{1,2,3,6,7,8,11,12,13\}}{15}=\frac{9}{15}
$$

Mediante el uso de clases residuales seria

$$
\begin{gathered}
B_{p} \cap C_{p} \\
\{\overline{1}, \overline{2}, \overline{3}, \overline{4}\} \cap\{\overline{0}, \overline{1}, \overline{2}, \overline{3}\}=\{\overline{1}, \overline{2}, \overline{3}] \\
\mathbb{P}\left(B_{p} \cap C_{p}\right)=\frac{\#\{\overline{1}, \overline{2}, \overline{3}\}}{5}=\frac{3}{5} \\
\mathbb{P}\left(B_{p} \cap C_{p}\right)=\frac{9}{15}=\frac{3}{5}
\end{gathered}
$$$$
\{\overline{1}, \overline{2}, \overline{3}, \overline{4}\} \cap\{\overline{0}, \overline{1}, \overline{2}, \overline{3}\}=\{\overline{1}, \overline{2}, \overline{3}\}
$$

Mediante el ejemplo anterior se permite evidenciar la equivalencia de las clases residuales al encontrar la probabilidad, es por ello su utilización en este caso.

$$
\mathbb{P}\left(B_{p}\right)=\frac{p-1}{p}=\frac{5-1}{5}=\frac{4}{5}=\mathbb{P}\left(C_{p}\right) \quad \wedge \quad \mathbb{P}\left(B_{p} \cap C_{p}\right)=\frac{p-2}{p}=\frac{5-2}{5}=\frac{3}{5}
$$

$$
\begin{gathered}
\rho_{p}(N)=\frac{\mathbb{P}\left(B_{p} \cap C_{p}\right)}{\mathbb{P}\left(B_{p}\right)^{2}}=\frac{\frac{p-2}{p}}{\frac{p-1}{p} * \frac{p-1}{p}}=\frac{\frac{p-2}{p}}{\frac{(p-1)^{2}}{p^{2}}}=\frac{p-2}{\frac{(p-1)^{2}}{p}}=\frac{p(p-2)}{(p-1)^{2}} \\
\frac{p(p-2)}{(p-1)^{2}}=\frac{p^{2}-2 p}{(p-1)^{2}}=\frac{p^{2}-2 p+1-1}{(p-1)^{2}}=\frac{(p-1)^{2}-1}{(p-1)^{2}}=\frac{(p-1)^{2}}{(p-1)^{2}}-\frac{1}{(p-1)^{2}} \\
=1-\frac{1}{(p-1)^{2}}
\end{gathered}
$$

Cuando p aumenta de tamaño $\rho_{\mathrm{p}}(N)$ Se aproxima a 1, es decir, la probabilidad es mucho más alta de la condición de independencia entre los sucesos "n no es múltiplo de $p$ " y " $N-n$ no es múltiplo de p". Se escogen dos números primos de tamaño semejante $\mathrm{p}_{1}, \mathrm{p}_{2}$ Tales que el primero es factor de $N$ y el segundo no lo es, la proximidad a dicha independencia es relativamente menor para $p_{2}$, pues para $p_{1}$ Seria $1+1 /(\mathrm{p}-1)$ Y para $p_{2}$ Tendrá ${ }^{1}{ }^{1 /}$ $(\mathrm{p}-1)^{2}$

$$
\begin{aligned}
& \text { Multiplicando (П: Producto) } \\
& \qquad \begin{array}{l}
\rho(N) \sim \prod_{p \leq \sqrt{N}} \rho_{p}(N) \sim \prod_{\substack{p \leq \sqrt{N} \\
p \mid N}}\left(1+\frac{1}{p-1}\right) * \prod_{\substack{p \leq \sqrt{N} \\
p \nmid N}}\left(1-\frac{1}{(p-1)^{2}}\right) \\
\text { Se Nombra } \\
\qquad P^{*}(N)=\prod_{\substack{p \leq \sqrt{N} \\
p \mid N}}\left(1+\frac{1}{p-1}\right) \quad y \quad P(N)=\prod_{p \mid N}\left(1+\frac{1}{p-1}\right)
\end{array}
\end{aligned}
$$

Estos dos productos son equivalentes debido a su acercamiento a la probabilidad máxima. Evidentemente $N$ tiene a lo mucho un factor primo $p^{*}$ Mayor que $\sqrt{N}$ Y por tanto.

$$
\begin{gathered}
P(N)=\prod_{p|N| N}\left(1+\frac{1}{p-1}\right)=\prod_{\substack{p \leq \sqrt{N} \\
p \mid N}}\left(1+\frac{1}{p-1}\right) *\left(1+\frac{1}{p^{*}-1}\right) \\
1 \leq \frac{P(N)}{P^{*}(N)}=\frac{\prod_{p \leq \sqrt{N}}\left(1+\frac{1}{p-1}\right) *\left(1+\frac{1}{p^{*}-1}\right)}{\prod_{\substack{p \leq \sqrt{N} \\
p \mid N}}\left(1+\frac{1}{p-1}\right)} \leq 1+\frac{1}{p^{*}-1} \\
1+\frac{1}{p^{*}-1} \leq 1+\frac{1}{\sqrt{N}-1} \rightarrow^{N \rightarrow \infty} 1 . \\
\text { Así mismo en el segundo producto } \prod_{\substack{p \leq \sqrt{N} \\
p \nmid N}}\left(1-\frac{1}{(p-1)^{2}}\right) \text { Se puede excluir la limitación } \\
p \leq \sqrt{N} .
\end{gathered}
$$

Por lo tanto, ahora 
Finalmente

$$
r_{2}(N) \sim \frac{N}{(\log N)^{2}} * \prod_{p \mid N}\left(1+\frac{1}{p-1}\right) * \prod_{p \nmid N}\left(1-\frac{1}{(p-1)^{2}}\right)
$$

La anterior formula puede reescribirse así:

$$
\begin{gathered}
r_{2}(N) \sim \frac{N}{(\log N)^{2}}\left(1+\frac{1}{2-1}\right) * \prod_{\substack{p|N| \\
p>2}}\left(1+\frac{1}{p-1}\right) * \prod_{p \nmid N}\left(1-\frac{1}{(p-1)^{2}}\right) \\
r_{2}(N) \sim \frac{2 N}{(\log N)^{2}} * \prod_{\substack{p \mid N \\
p>2}}\left(1+\frac{1}{p-1}\right) * \prod_{p \nmid N}\left(1-\frac{1}{(p-1)^{2}}\right)
\end{gathered}
$$

E1 primer producto recorre todos los primos impares, ya que $p>2$, entonces

$$
\begin{gathered}
1-\frac{1}{(p-1)^{2}} * 1+\frac{1}{p-2}=1+\frac{1}{p-1} \\
r_{2}(N) \sim \frac{2 N}{(\log N)^{2}} * \prod_{\substack{p|N| N \\
p>2}}\left(1+\frac{1}{(p-1)^{2}}\right)\left(1+\frac{1}{p-2}\right) * \prod_{p \nmid N}\left(1-\frac{1}{(p-1)^{2}}\right) \\
r_{2}(N) \sim \frac{2 N}{(\log N)^{2}} * \prod_{\substack{p|N| N \\
p>2}}\left(1+\frac{1}{(p-1)^{2}}\right) \prod_{p \nmid N}\left(1-\frac{1}{(p-1)^{2}}\right) * \prod_{\substack{p|N| N \\
p>2}}\left(1+\frac{1}{p-2}\right) \\
r_{2}(N) \sim \frac{2 N}{(\log N)^{2}} * \prod_{2<p<N}\left(1+\frac{1}{(p-1)^{2}}\right) * \prod_{\substack{p \mid N \\
p>2}}\left(1+\frac{1}{p-2}\right)
\end{gathered}
$$

Ejemplo. Sea $n:=12$

$p \mid n$ serian 2 y 3 , entonces

$$
\begin{gathered}
\prod_{p \mid N}\left(1+\frac{1}{p-1}\right)=\left(1+\frac{1}{2-1}\right) *\left(1+\frac{1}{3-1}\right)=1+\frac{1}{1} * 1+\frac{1}{2} \\
=2 * \frac{3}{2}
\end{gathered}
$$

$p \nmid N$ seria 5,7 y 11

$$
\begin{gathered}
\prod_{p \nmid N}\left(1-\frac{1}{(p-1)^{2}}\right)=\left(1-\frac{1}{(5-1)^{2}}\right) *\left(1-\frac{1}{(7-1)^{2}}\right) *\left(1-\frac{1}{(11-1)^{2}}\right) \\
\prod_{p \nmid N}\left(1-\frac{1}{(p-1)^{2}}\right)=\frac{15}{16} * \frac{35}{36} * \frac{99}{100}
\end{gathered}
$$

Para la formula reescrita seria

$$
\prod_{2<p<N}\left(1+\frac{1}{(p-1)^{2}}\right) * \prod_{\substack{p \mid N \\ p>2}}\left(1+\frac{1}{p-2}\right)
$$

Para $p:=3$ como esta en ambos productos, se tiene que

$$
\begin{aligned}
\left(1-\frac{1}{(p-1)^{2}}\right) *\left(1+\frac{1}{p-2}\right) & =\left(1-\frac{1}{(3-1)^{2}}\right) *\left(1+\frac{1}{3-2}\right) \\
=\frac{3}{4} * 2 & =\frac{6}{4}=\frac{3}{2}
\end{aligned}
$$

Para $p:=5$ como esta en un solo producto

$$
\left(1-\frac{1}{(p-1)^{2}}\right)=\left(1-\frac{1}{(5-1)^{2}}\right)=\frac{15}{16}
$$

Para $p:=5$ como esta en un solo producto

$$
\left(1-\frac{1}{(p-1)^{2}}\right)=\left(1-\frac{1}{(7-1)^{2}}\right)=\frac{35}{36}
$$

Para $p:=11$ como esta en un solo producto

$$
\left(1-\frac{1}{(p-1)^{2}}\right)=\left(1-\frac{1}{(11-1)^{2}}\right)=\frac{99}{100}
$$

De esta manera probabilística se ha hallado una probable expresión muy próxima para la cantidad de representaciones de un número par $\mathrm{N}$ como suma de dos primos. La fórmula ha sido verificada por extensos métodos computacionales.

\section{Godfrey Harold Hardy y John Edensor Littlewood}

\section{El método de círculo}

En 1923 Godfrey Harold Hardy y John Edensor Littlewood desarrollaron el método del círculo, usado por Hardy y Rāmānujan para estudiar de cuantas maneras distintas se puede expresar un número como sumas de otros números (Cantidad de Particiones)

Este método es una de las herramientas más apropiadas en la Teoría Aditiva de Números para emplear en aquellos problemas que buscan calcular la cantidad de representaciones de un número $\mathrm{n}$ como suma de otros números.

Sea cualquier subconjunto de los enteros positivos $S \mathrm{c} N$ y un numero fijo k de sumandos se 
pretende encontrar el orden de magnitud de la función $\mathrm{r}(\mathrm{n} ; \mathrm{k} ; \mathrm{S}): \mathrm{N} \rightarrow \mathrm{N}$ definida como

$$
r(n ; k ; \mathcal{S}):=\#\left\{\left(a_{1}, \cdots, a_{k}\right) \in \mathcal{S}^{k} \mid n=a_{1}+\cdots+a_{k}\right\}
$$

Que cuenta con el número de representaciones de $\mathrm{n}$ con $\mathrm{k}$ sumandos del conjunto $\mathrm{S}$.

Ejemplo. Sea $\mathrm{S}=\{1,2,3,4,5\}$ Con $n=8$ y $k=2$

Entonces dado el subconjunto $\mathrm{S}$ contenido en los naturales $(S c N)$ se busca representar a 8 como suma de tres números de este subconjunto.

Las parejas serian:

$$
\begin{aligned}
& (5,3)=5+3=8 \\
& (3,5)=3+5=8 \\
& (4,4)=4+4=8
\end{aligned}
$$

Ahora aplicamos

$$
\begin{gathered}
r(8 ; 2 ; \mathcal{S})=\#\{(5,3),(3,5),(4,4)\} \\
r(8 ; 2 ; s)=3
\end{gathered}
$$

Nombrando $P:=\{p: p$ es un número primo $\} \mathrm{Y}$ $\mathrm{R}\left\{n^{r}: n \in \mathrm{N}\right\}$ Para un exponente entero $\mathrm{r}$ fijado, por ejemplo;

Si $S=N$ y k no está fijo, el problema es la cantidad de representaciones que pueda tener dicho numero como suma de otros.

- Si $S=P$ y $k=2$ el objeto del estudio es la suma de dos primos $P+P$, donde ocupa un lugar destacado la Conjetura de Goldbach, pro también se trataría la condición $P+P=$ impar.

- Si $S=P$ y $k=3$ se busca investigar $P+P+P$ donde el Teorema de Vinogradov confirma que todo número impar lo suficientemente grande esta en este conjunto.

- Si $\mathrm{S}=\mathrm{R}$ y se fija un $\mathrm{k}$ se quiere analizar $R+\ldots+R$ ( $k$ veces), se conoce como Problema de
Waring busca las formas de expresar un numero entero positivo como la suma de $k$ potencias $r$-ésimas.

\section{Conclusiones y recomendaciones}

A finales del siglo XX y principios del presente, las conjeturas emblemáticos de la matemática se les ha dado una demostración apoyada, aunque no sea del agrado de algunos, en la informática, para mencionar solo las dos de mayor difusión la conjetura propuesta por Francis Guthrie en 1852 sobre los colores mínimos para colorear un mapa que da origen entre otros a la topología y en particular a la teoría de grafos y demostrada a mediados de los 70 por Kenneth Appel y Wolfgang Haken con el apoyo de un programa informático, el denominado último teorema de Fermat, hoy teorema de FermatWiles, nace en la Grecia clásica y es conjeturado por Pierre de Fermat en 1637 después de aportes de grandes matemáticos como Euler, Legender, Lamé, Kummer, Dirichiert por fin Andrew Wiles en 1995 después de algunos contratiempos da una demostración aceptada por la mayoría del mundo matemático.

La conjetura que motivo este trabajo con raíces que se pierden en el tiempo y conjeturada 1792, ha sido, igual que las anteriores mencionadas, atacadas desde lo finito utilizando, con cada vez más eficaces algoritmos computacionales y desde las más variadas ramas de la matemática. Aquí se presentaron intentos de demostración a partir de la teoría de números primos, en la búsqueda de fórmulas que describan todos los primos, intento que nunca se ha logrado concretar. Demostraciones que echan mano de la probabilidad con que aparecen los números primos, que nuevamente son esquivos a dejarse encasillar. Se echó mano a la lógica en otra de los intentos aquí presentados, nuevamente sin el éxito esperado. Divide y vencerás tal vez pensó Ricardo G. Barca en el intento que aquí se presenta, pero se le escapó alguna parte. Siguiendo la idea de Polya, se trata de solucionar siguiendo el camino 
que ha dado solución a otros problemas mediante un acercamiento heurístico, pero sin lograrlo.

\section{Referencias}

Barca Ricardo. (2008). La Conjetura de Goldbach. Universidad Tecnológica Nacional, Argentina.

Bravo, César \& Ojeda Uresti, Juan. (2006) ¿Es la conjetura de Goldbach?, Revista de cultura científica de la facultad de ciencias de la Universidad Nacional Autónoma de México.

Euler, Leonard (1742). Carta de respuesta a Christian Goldbach. Matematico y fisico suizo.

Goldbach, Christian. (1742). Carta a Leonhard Euler. Matemático de Prusia.

Guevara Bravo, J. César y Ojeda Uresti, Juan. (2006). ¿Formuló Goldbach la conjetura de Goldbach? Ciencias 81, enero-marzo. p. 74.

Gonzales, E. (2013). Acerca del estado de la cuestión o sobre un pasado reciente en la investigación cualitativa con enfoque hermenéutico. Unipluriversidad, 13(1), 60- 63.

Hernández, I. (2012). Investigación cualitativa: una metodología en marcha sobre el hecho social. Revista Rastros Rostros, 14(27), 57-68.

Jiménez, Rafael, Gordillo, Jorge \& Rubiano, Gustavo. (2004) Teoría de Números para principiantes. Bogotá: Pro-offset. p. 1

Jiménez, Rafael, Gordillo, Enrique \& Rubiano, Gustavo. (2004). Teoría de Números para principiantes. $2^{\mathrm{a}}$ edición. Facultad de ciencias. Universidad Nacional de Colombia. Colombia. pág. 75.

Newton, Isaac. (1676). Carta a Robert Hooke. Matemático inglés.

Niño Rojas, Francisco. (2006). Sobre la Conjetura de Goldbach. Universidad Industrial de Santander Colombia. Recuperado de http:// repositorio.uis.edu.co/jspui/bitstream /123456789/7247/2/119393.pdf.

Peral Manzo, Mario. (2007). Replanteamiento de la Conjetura de Goldbach. Universidad Pedagógica Nacional, México.

Rueda, M. (2007). La investigación etnográfica y/o cualitativa y la enseñanza en la universidad. Revista Mexicana de Investigación Educativa, 12(34), 1021-1041.

Tesoro, Rafael. (2011). Sumando primos ¿Hay tres sin dos? Universidad Autónoma de Madrid, España. Recuperado de http://www.uam.es/ personal_pdi/ciencias/fchamizo/posgrado/ mastertesoro.pdf.

Vinogradov, Iván. (1937) El Teorema de Vinogradov. Rusia.

Zaldivar, Felipe. (2006). Introducción a la teoría de grupos. Sociedad matemática Mexicana México: Reverte. p. $6-12$. 